\title{
Notas sobre los textos del ataúd de Esoeris en Montevideo: prosopografía
}

Víctor Capuchio" y Diego M. Santos**

Recibido:

28 de abril de 2019

Aceptado:

9 de diciembre de 2019

\section{Resumen}

Los autores presentan una nueva lectura de las inscripciones del ataúd de Esoeris, hija de Nespamay, junto con una breve descripción del mismo y una discusión respecto de las citadas inscripciones. La intención principal del artículo es ofrecer una transcripción del nombre de la madre de Esoeris, corrigiendo los datos prosopográficos que brinda el ataúd.

Palabras clave

Ataúd egipcio Akhmim

Textos funerarios

Egipcio clásico

Prosopografía

\section{Notes on Esoeris coffin texts in Montevideo: the prosopography}

\begin{abstract}
In this paper, we present a new interpretation of the inscriptions on the Esoeris' coffin, daughter of Nespamay, based on a brief description of the coffin and a discussion of the named inscriptions. The main aim of this paper is to present a transcription of the name of Esoeris' mother and correct the prosopographical data offered by this coffin.
\end{abstract}

Keywords

Egyptian coffin

Akhmim

Funerary texts

Classical Egyptian

Prosopography

\footnotetext{
* Museo de Historia del Arte (MuAr), Intendecia de Montevideo. Ejido 1326, Palacio Municipal (CP 1120009 Montevideo, Uruguay. E-mail: vcapuchio@gmail.com

** Departamento de Humanidades y Arte, Universidad Pedagógica Nacional (UniPe). Piedras 1080 (C1070AAV)

Buenos Aires, Argentina. E-mail: diego.santos@unipe.edu.ar
} 
1. Las referencias a TM Nam TM Per remiten a la base de datos de Trismegistos, que puede ser consultada en https://www. trismegistos.org/ref/index (acceso: 21 de octubre, 2019).

2. 3s.t-wr.t "Isis la grande", Ranke (1935), nombre bien atestiguado en Época Ptolemaica, Lüddeckens (1980), TM Nam 190.

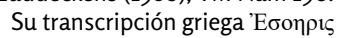

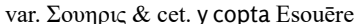
sugieren la reconstrucción *əs'wērə (seguimos aquí el sistema clásico descripto en Loprieno, 1995).

3. Para una introducción rápida al contexto, ver Elias y Lipton (2008) y bibliografía allí citada. Véase también el clásico Gauthier (1931).

4. En la base de datos trismegistos. org el ataúd de Montevideo lleva el número TM Ref 89276. Su madre es TM Per 162714, leyendo su nombre $t 3$-šr.t-mnw, sin aportar argumentos. Se trataría, entonces, de una persona fantasma. Nótese, por otra parte, que la Esoeris de Montevideo es la única de ese nombre hija de un Nespamay repertoriada.

\section{Introducción}

El museo de Historia del Arte de Montevideo (República Oriental del Uruguay) hospeda una pequeña colección de piezas egipcias que, sumadas a un conjunto de calcos, dan testimonio, a través de la investigación, conservación y presentación museográfica de las mismas, de un importante legado en el Río de La Plata.

El punto central de la actual exhibición se ubica alrededor del conjunto arqueológico conformado por el ataúd de un individuo femenino llamado $^{1}$ Esoeris $^{2}$ (TM Per 59797) y sus restos momificados. Una máscara está asociada a los mismos desde el momento de su donación a fines del siglo XIX, pero su relación con el grupo anterior - si hay alguna- es dudosa (ver abajo).

Desde hace más de cuatro décadas se han realizados estudios multidisciplinarios que nos aportan valiosos datos de quien en vida habría sido una sacerdotisa que cumplía funciones como tañedora del sistro en el templo del dios Min en la ciudad de Akhmim ${ }^{3}$ en el Alto Egipto (Bianchi, 1993; Castillos, 1976, 1980, 2001, 2005). Era hija del estolista Nespamay (Ns-p3-m3j) [TM Per 162713] y de una mujer llamada Taamon (T3-jmn, véase abajo), también tañedora del sistro de Min. El propósito de esta breve colaboración es publicar una nueva versión de las inscripciones del ataúd y una nueva lectura del nombre de la madre de Esoeris ${ }^{4}$.

Como es sabido desde las publicaciones pioneras de J. Castillos señaladas, a finales del siglo XIX el conjunto fue adquirido en el Museo de Būlāq por un ingeniero uruguayo de nombre Luis A. Viglione (1852-1891), quien visitó Egipto en 1889 (Salem, 2018; Viglione, 1890). Es difícil establecer lo sucedido con su colección, por las escasas referencias al respecto. Una parte de ella fue donada por su viuda Delfina Labarriere (1859-1934) en 1896 al Museo Público de Buenos Aires y hoy se conserva en el Museo Etnográfico de la Facultad de Filosofía y Letras de la Universidad de Buenos Aires (Santos, 2003). De las donaciones en vida practicadas por el ingeniero, tenemos registradas tres: un grupo de calcos al municipio de La Plata (ahora en el Museo de La Plata; Daneri, 1981) y, probablemente, la pequeña momia defectuosa del Museo de La Plata (Santos, 2012).

La donación de la momia de Esoeris, su ataúd, una máscara y un calco en yeso la conocemos por nota del Ministerio de Instrucción Pública del 16 de Noviembre de 1889 dirigida al director del Museo Nacional donde se menciona la comunicación del Ing. Viglione con dicho ministerio, con la intención de donar una "momia con su sarcófago" y "la estatua en yeso del rey Chefrén" que había adquirido en el "Museo de Bulak". Esta donación se encuentra transcripta en carta dirigida por el director del Museo de Historia Natural C. Diego Legrand (14/07/1955) al nieto del donante, Fausto Viglione, que confirma dicha nota y menciona que "desgraciadamente la estatua de yeso no ha llegado hasta nosotros pero si la momia, el sarcófago y una mascarilla dorada" (correspondencia en poder de la tataranieta de Luis A. Viglione, Andrea Viglione, a quien agradecemos las referencias).

La intención de donarla al estado uruguayo debe haber sido la causa de su adquisición, puesto que escribe al gobierno oriental al poco tiempo de regresar a Egipto. Tanto el calco de la estatua como ambas momias (la de Montevideo y la de La Plata) son mencionadas en su carta VIII, escrita desde El Cairo el 15 de Mayo de 1889 y reproducida en Viglione (1890).

Una vez en Montevideo, el nuevo director del Museo de Historia Natural, Carlos Berg (1843-1902), con el fin de exponer la reciente adquisición al público, le practicó un corte longitudinal a lo largo de todo el cuerpo, retirando los vendajes y dejando expuesto el lado izquierdo del mismo y su cabeza. En los años '70 se realizaron estudios 
radiológicos, se identificó la madera del ataúd, se estudiaron los pigmentos de las pinturas y se efectuaron traducciones de los textos (Castillos, 1976).

En el año 2001, ante el inminente cierre del mencionado Museo, se concretó el préstamo del conjunto funerario al Museo de Historia del Arte y se planificó una batería de exámenes sobre el mismo. Entre 2002 y 2004 se le realizaron estudios de tipo egiptológico, bacteriológicos, radiológicos, antropométricos, odontológicos, datación por radiocarbono, identificación de la especie vegetal del ataúd, extracción de muestras de ADN y reconstrucción facial de la momia utilizando técnicas forenses (Capuchio, 2004; Castillos, 2005). Por último en 2016, se le practicó una tomografía que arrojó más información sobre el proceso de momificación y el cuerpo de Esoeris (Capuchio, 2016).

En el año 2004 se contó con la colaboración de la Lic. Laura del Puerto y el Lic. Hugo Inda, integrantes del Laboratorio de Estudios del Cuaternario de la Facultad de Ciencias, con el objetivo de poder identificar la especie vegetal de la madera que fue utilizada para la confección del ataúd de Esoeris. De acuerdo a un procedimiento de observación al microscopio, se recabaron las características anatómicas macro y microscópicas de la muestra leñosa y se efectuaron cortes de los tres planos del leño, que fueron subsecuentemente procesados; los datos relevados fueron introducidos y contrastados en bases de datos digitales globales sobre especies leñosas. Esto permitió la identificación de la muestra como Ficus Sycomorus (Moraceae), la madera más común usada en la confección de ataúdes prácticamente en toda la historia egipcia (Nicholson y Shaw, 2000), lo que posibilitó confirmar los estudios de Castillos $(1976,1980)$, quien además observó un segundo tipo de madera en las clavijas, aún sin identificar.

\section{Datación}

La datación del ataúd es problemática. Las edades radiocarbónicas publicadas (Bracco y Ures, 2002; Capuchio, 2003; Castillos, 2005) lo ubican entre la segunda mitad del siglo V a.C. y la primera mitad del siglo IV a.C. No podemos descartar un margen de incertidumbre con respecto a la fiabilidad de estas edades (al respecto véase Cockitt, Martin y David, 2014). Esta datación resulta bastante temprana para las características estilísticas del ataúd, que es claramente ptolemaico ${ }^{5}$.

En 2013, Ruth Brech estableció la tipología, secuencia y datación de los ataúdes procedentes de Akhmim en su obra Spätägyptische Särge aus Akhmim (Brech, 2013). En ésta incluyó el ataúd montevideano con la signatura E b 3 (Brech, 2013). Este grupo $\mathrm{E} b$ está integrado por cuatro ataúdes claramente relacionados. Es un grupo que presenta problemas en cuanto a su datación: puede demostrarse que es ptolemaico (como todo el conjunto E) pero no hay evidencia prosopográfica ni de otro tipo - al menos hasta ahora- que permita ubicar a estas personas en un momento determinado de este período, por lo que Brech (2013) concluye que pueden ser de cualquier momento entre el inicio y el final del mismo. Para todo el conjunto E asigna, con cautela, una datación entre los siglos III-II a.C. (Brech, 2013). En este contexto, quizás deban tenerse en cuenta los estudios de radiocarbono mencionados para sugerir una datación en el siglo IV a.C., posibilidad que debería ser explorada en el futuro.

Si el vínculo, especulativo pero posible, que propone Castillos con el conjunto del Ägyptisches Museum Berlin, Inv. 31212+31213 (Castillos, 2001, 2005) (E c 5-6 en Brech, 2013) es cierto, habría un puente entre el grupo Eb y el grupo Ec, con una datación de este último posterior al siglo III a.C. y, probablemente, de mediados del período ptolemaico. No obstante y considerando lo atractivo de esta solución, no hay elementos para confirmar tal relación y especialmente, como señaló Brech (2013), no sabemos de cuál $N s-p 3-m 3 j$ de esa genealogía podría tratarse. El antiguo ocupante del ataúd es hijo de $N s-h r w$, nieto de
5. Datación sobre la que actualmente hay acuerdo en la comunidad científica sobre ataúdes de estas características, especialmente luego de la obra de Brech, véase abajo. Dataciones previas los ubican en la dinastía XXVI (Castillos, 1976, sostenida en la bibliografía en uso en aquel entonces; Bianchi, 1993), datación que sostiene en Castillos (2001). El mismo siguió posteriormente los estudios de radiocarbono y parece adoptar la datación en el siglo $\mathrm{V}$ a.C. (Castillos, 2005). 
6. Las máscaras ptolemaicas tardías se consideran en Grimm (1974), a cuya discusión remitimos. Véase también, sobre el sentido general, y cambios, de las máscaras de esta época y el uso de láminas de oro, Stadler (2001). La misma opinión tenían J. R. Ogdon (citado en Castillos, 2001, 2005) y A. Bianchi (1993). Brech (2013) también considera probable que la procedencia de la máscara sea diferente a la del resto del conjunto.
$N s-p 3-m 3 j$ y bisnieto de $H r-n(j) s w(. t)$. Su madre, tañedora del sistro de Min, se llamaba $P 3=s$-hrj-ntrj (Brech, 2013, lee el nombre del bisabuelo como $h r-r s n t)$. De este personaje se conserva una estela funeraria en el Bowdoin College, con la misma genealogía, ver de Meulenaere, Limme y Quaegebeur (1985). Es decir, si hubiera alguna relación familiar, la Esoeris de Montevideo podría ser hija o tía del Nespamay de Berlín.

Aún más interesante resulta la máscara por las posibilidades de datación que ofrece. Aunque la tipología de las máscaras ptolemaicas está por hacerse (en este sentido, Miatello, 2012), en este caso suele considerarse de época ptolemaica tardía con bastante seguridad ${ }^{6}$. No tenemos forma alguna de saber si la máscara pertenecía a Esoeris o si es un agregado al conjunto. Sólo sabemos que procede de la misma donación. Si fuera lo primero, daría una fecha relativamente tardía para el ataúd, posterior al siglo II a.C., la cual consideramos improbable.

\section{El ataúd y sus inscripciones}

El ataúd es antropomorfo y representa la momia de pie y sobre un pedestal. Está conformado por dos partes, una tapa abovedada y una base que se juntan a través de seis clavijas aferradas en la base (Figura 1). La superficie del ataúd tiene una capa de estuco donde se aplicaron los colores en ambas partes a excepción de la parte trasera del cubeto. El interior de ambas partes no está ni decorado ni coloreado (elemento no exclusivo, pero sí característico en otros de similar tipología), aunque sí presenta una capa muy fina de estucado en la cubeta. Este revestimiento se ha ido desprendiendo con el tiempo y en 2016 fueron extraídas muestras para un estudio químico de su composición.

La decoración del ataúd es la característica del grupo E b descripto por Brech (2013). El rostro representado en el ataúd está rodeado por una peluca tripartita de color azul y recubierto con hojas de oro (Stadler, 2001), resaltando el gran tamaño de sus ojos y cejas que están delineadas en negro. Entre las dos partes frontales de la peluca, que caen a ambos lados de la cara y sobre el pecho, se extiende una decoración de líneas horizontales de colores azul (10), rojo (5) y verde (4) alternados en ese orden y en un fondo naranja oscuro. Cubriendo el pecho un gran collar semicircular wsh formado por 16 filas con diferentes diseños en las cuentas que lo conforman: figuras geométricas (círculos, triángulos y elipses) o flores de loto en la tercera, novena y decimocuarta hilera. El collar termina por debajo de los hombros y en sus extremos tiene grandes cabezas de halcón coronadas por un disco solar.

Un segundo registro casi desaparecido por el desprendimiento del estucado, se ubica más abajo del collar y a la altura del abdomen. Está dominado por la representación de la diosa Nut alada con un disco en su cabeza con sus extremidades abiertas y conformadas por tres hileras de plumas, la primera roja, una segunda de color verde y la inferior de color azul, que protegen el cuerpo de la difunta representada en el registro inferior. Arriba de cada una de sus alas, un ojo-w $\underline{\text { d }}$.t. Hay una cartela con texto perdido. A la derecha y a la izquierda se ubican los cuatro compartimentos para los hijos de Horus, pudiendo reconocerse - en el estado actual- sólo a Qebehsenuf en posición inferior a la derecha de la difunta.

El registro siguiente y más abajo del anterior, nos muestra el cuerpo momificado de Esoeris que descansa sobre una cama zoomorfa, con patas y cabecera de león. Entre las patas de la cama se representaron los vasos canopos que resguardan los órganos de la difunta, con cabezas de los cuatro hijos de Horus en este orden: Imset, Hapy (perdido), Duamutef y Qebehsenuf. En los laterales de esta imagen encontramos divinidades identificadas en sus respectivos textos cursivos (a saber izquierda Isis, derecha Neftis) enmarcadas por inscripciones (Figuras 2 y 3), ambas lamentándose por su hermano Osiris, identificado con la difunta. Detrás de cada diosa, hay sendas inscripciones que parecen mencionar a Anubis. Las cuatro inscripciones, cada una en tres columnas, 


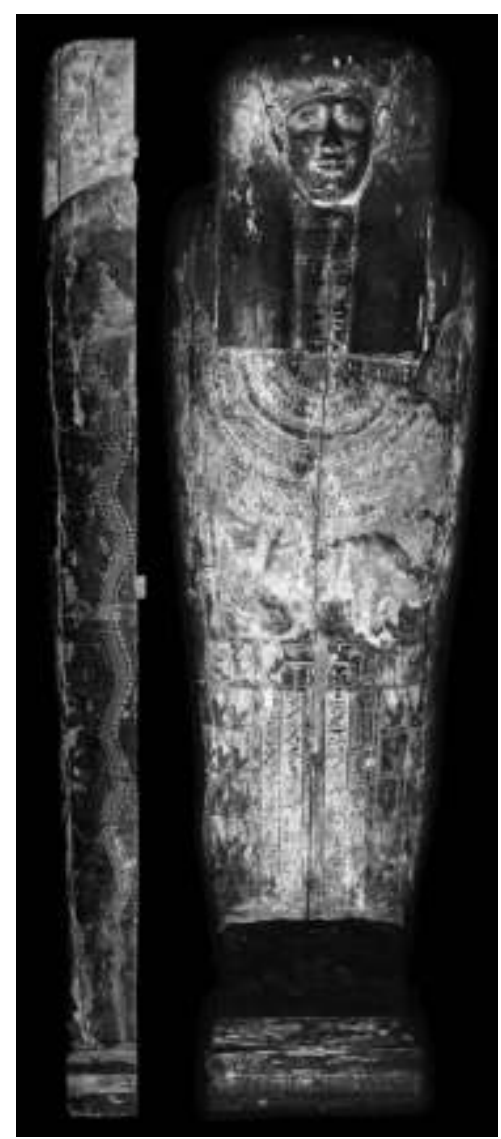

Figura 1. Ataúd de Esoeris vista frontal y lateral. Fotografía Víctor Capuchio.

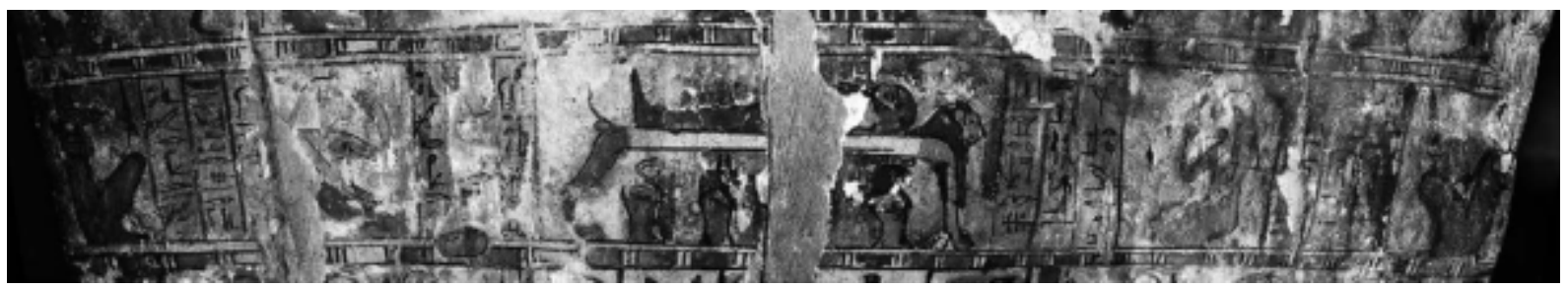

Figura 2. El registro donde vemos el cuerpo momificado de Esoeris que descansa sobre una cama zoomorfa. En ambos laterales de esta imagen encontramos cuatro textos. Fotografía Víctor Capuchio.

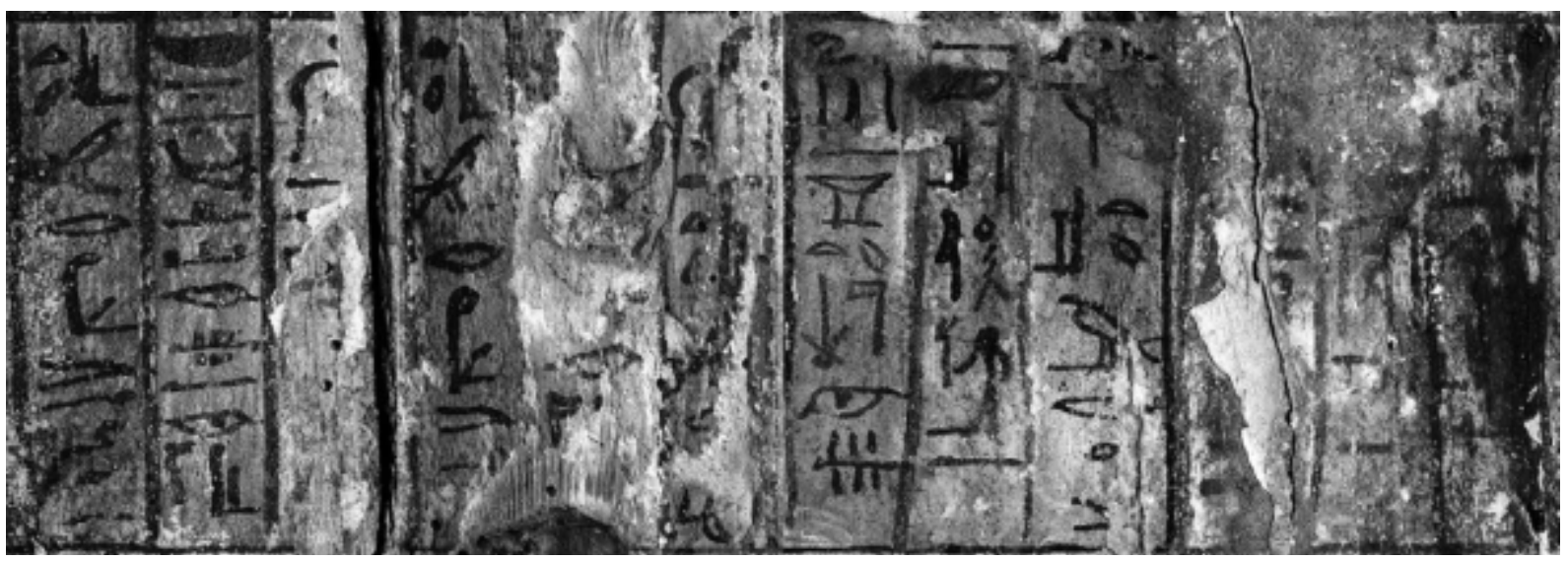

Figura 3. Comparación sinóptica de los cuatro textos que se observan en la Figura 2. Fotografía Víctor Capuchio. 
7. Por supuesto, no la difunta sino el nombre de la diosa Isis con su epíteto wr.t "grande" (ver Leitz, 2002). presenta la estructura siguiente: $\underline{d} d m d w$ jn [nombre de divinidad + epíteto] jr $s^{3} n$ Wsjr [nombre del difunto] $m)^{\top}(. t)$-hrw d.t.

Izq. A: ${ }^{1} \underline{d} d m d w$ jn $3 s . t$ wr.t $[.]^{2}[\ldots]$ Wsjr ${ }^{3} 3 s . t-w r . t m{ }^{\complement}(. t)^{\mathrm{A}}-[h r w \underline{d} . t]$. "Palabras dichas por Isis la Grande ${ }^{7}[\ldots]$ Osiris Esoeris justificada eternamente".

Notas:

A La escritura de $m 3^{\top} . t-h r w$ aquí y en toda sus apariciones en este ataúd es Aa 13 (o A 15) + P 8, lit. $m$ hrw , reflejando la pronunciación contemporánea, véase el nombre

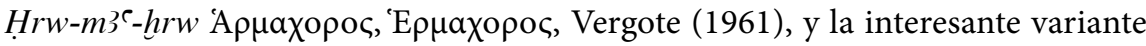
jeroglífica $H r w-m-m{ }^{\complement}-h r w$, donde compromete le pronunciación con la ortografía arcaizante del nombre (Vernus, 1978; para los cuatro nombres, v. TM Nam 269). Aquí leemos, por convención, todas las apariciones como $m 3^{\top}(. t)-h r w$.

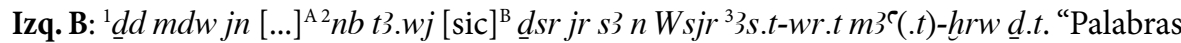
dichas por [...] señor de Tadjeser: protejo a Osiris Esoeris justificada eternamente".

Notas:

A Por el título $n b t 3 \underline{d} s r$ debería tratarse de Anubis pero los trazos parecen corresponder a wsjr "Osiris". Probablemente, error del escriba (ver Leitz, 2002).

B La ortografía $n b t 3 . w j$ en lugar de $n b t 3$ es esperable e intenta reflejar la pronunciación contemporánea del compuesto, que resultaba homófono de su antigua forma dual: $n b-t 3 *$ nib-'ta3 > nəb'ta / nb- $t$ 3.wj *nib-ta3wvj > nəb'ta.

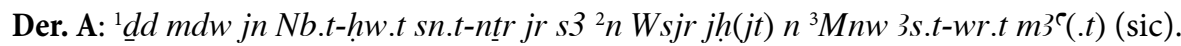
"Palabras dichas por Neftis, la hermana del dios: protejo a Osiris tañedora del sistro de Min Esoeris, justificada”.

Der. B: ${ }^{1}[\underline{d} d d m d w \text { jn Jnpw }]^{\mathrm{A}}[\ldots]^{2}[\ldots h n t j]^{\mathrm{B}} \operatorname{sh-ntr}[\ldots]{ }^{3} \mathrm{j} r s^{3} n$ Wsjr [...]. “[Palabras dichas por Anubis...] [... señor] de la Tienda del Dios [...]: protejo a Osiris [...]”.

\section{Notas:}

A Se reconocen trazos de la escritura de este título.

B El título vinculado a la 'Tienda del dios' no deja dudas de que se trata de Anubis, de cuyo nombre pueden reconocerse algunos trazos remanentes, ver Leitz (2002).

Enmarcando ambos registros y la inscripción central hay catorce grupos que suman 42 divinidades armadas con cuchillos (algunas perdidas): los jueces del capítulo 125 del Libro de los Muertos.

Siguiendo la línea media del ataúd, entre las piernas de Esoeris encontramos el texto principal conformado por cinco columnas de inscripciones jeroglíficas cursivas que se leen de arriba a abajo y de derecha a izquierda del observador (Figura 4). Las inscripciones fueron leídas por A. Erman (Castillos, 2001) en los albores del siglo pasado y por J. Capart en 1936, traducidas y estudiadas por primera vez por J. Castillos (1976, retomadas en las subsiguientes publicaciones) y releídas por A. Bianchi en 1993. Ofrecemos aquí una nueva traducción que creemos presenta algunos aportes a la discusión.

Las cinco líneas frontales del ataúd de Esoeris presentan una variante de uno de los Textos de Nut, fórmula 5, parte 3 a-b (en la lista de Elias, 1993), ya presente en los Textos de las Pirámides (TP $368+588$, para una referencia rápida, Allen, 2005), pero circula desde el Reino Medio de forma independiente (Galán, 2013), por lo que no se 


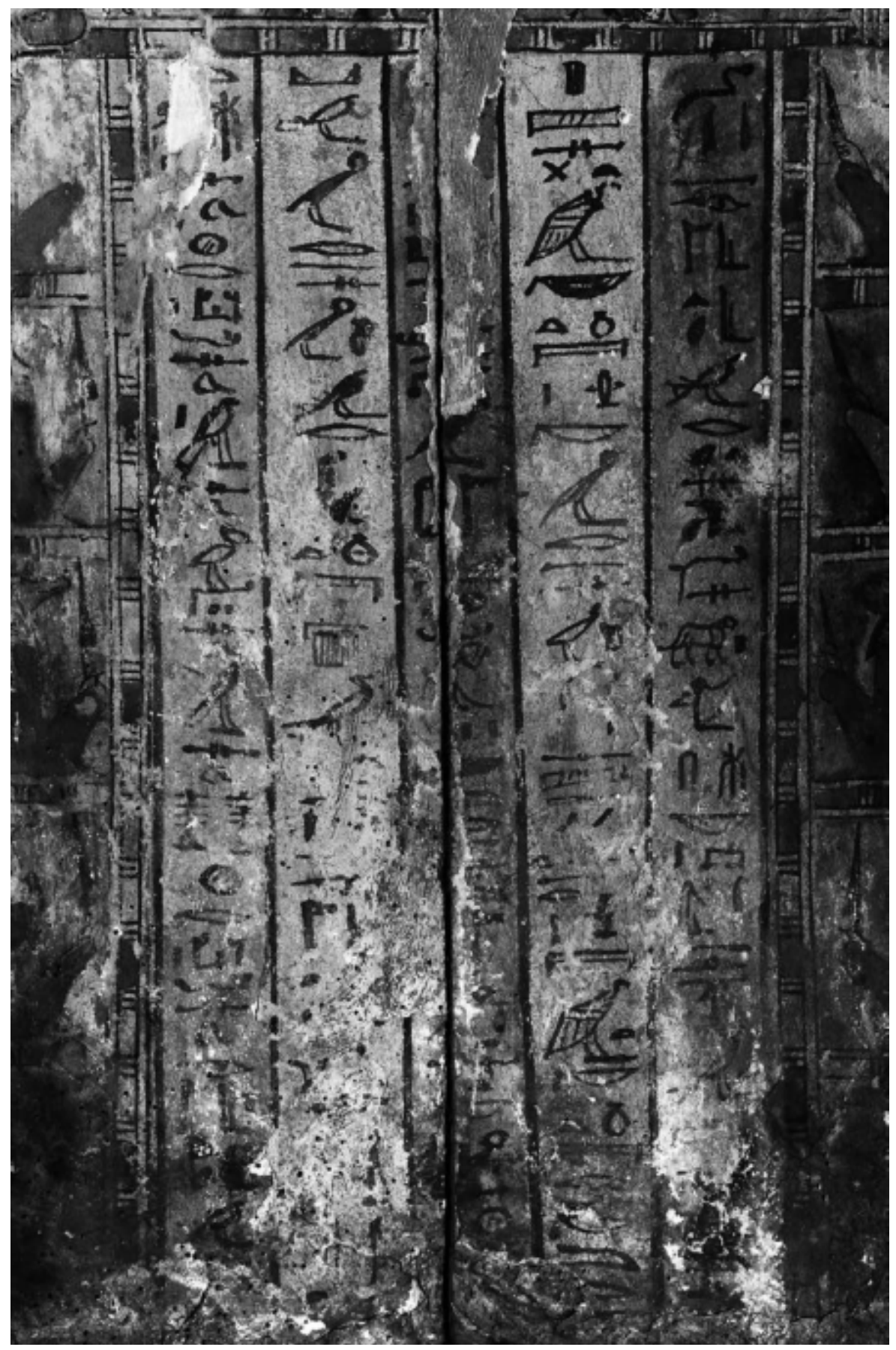

Figura 4. Texto principal en la línea media del ataúd entre las piernas de Esoeris. Fotografía Víctor Capuchio.

trata necesariamente de una cita sino de la circulación, re-elaboración y re-lectura de parte de un texto por fuera del primer corpus donde se lo encuentra. Está seguida de una combinación de elementos de TP 25, 447, 450 y 568 y no simplemente de TP 25. Sobre este texto en el mencionado ataúd de Nespamay en Berlín, afirma:

Contra Brech (p. 217), the text of D 4, lines 5 (end) - 7 is not simply PT Spell 447, but rather a combination of related TP Spells 25, 447, 450 and 568. The begining invoking 'someone', Horus, Thoth, Dewen-anwy and Osiris "having gone for his 
ka" follows PT 25, while the use of the preposition $h r$ for 'to' follows the later spells.

(Ritner, 2016, p. 184, n. 10).

El texto, en forma similar a como aparece en el ataúd, se encuentra como el No 5 en el volumen III de $s 3 h w$ (conocida por el pWalters Art Museum 551 y el pBM 10081, ambos tardíos-ptolemaicos, ver Assmann, 1990, 2008; Barbash, 2011). Se trata de parte de un rito de la práctica litúrgica calendárica osiriana reapropiado en contexto funerario en virtud de la identificación del difunto con la divinidad (Barbash, 2011). El escriba del ataúd montevideano debe haber usado otra redacción: por ejemplo, donde dice en c. 2 $s \check{s} t 3-n(j)-h r j$ (reinterpretación del epíteto de Nut, š.t-p.t, en los Textos de las Pirámides, ver Leitz, 2002b), los pWalters 551 y pBM 10081 dicen $s \check{t}$ t3-p.t, distinción que debe haber surgido de la lectura de un texto donde - cielo- (volcado por dos homónimos) se encontrase escrito solo con el signo $\mathrm{N} 1$.

La elección de este texto frontal es una característica frecuente de los ataúdes ptolemaicos de Akhmim (Brech, 2013). La lectura sinóptica de la versión de Esoeris con la que aparece en los ataúdes de Nespamay en Berlin (Ägyptisches Museum Inv.-Nr. 31.212-31.213) ambas presentan errores - sugiere una redacción común en Akhmim, ubicada a mano de quienes elegían el programa textual y producían los ataúdes ptolemaicos descubiertos en las necrópolis de dicha ciudad. Sólo un estudio del texto en todos los ataúdes conocidos de las necrópolis de esta ciudad - aún por hacerse - podría revelar una edición local de este grupo, como sucede con el Libro de los Muertos en Akhmim en época ptolemaica (Mosher, 2001, 2002, 2016; Scalf, 2014, 2017).

La escritura de memoria, en virtud de los errores, no puede ser descartada. Hemos usado esta versión y la del ataúd de Nespamay de Berlín para establecer la lectura del de Esoeris.

(c. 1) $d d m d w$ jn Wsjr 3s.t-wr.t $s 3(. t)^{\mathrm{A}} s m 3 . t j^{\mathrm{B}} N s-p 3-m 3 j \operatorname{ms}(. n) n b(. t)-p r j h j . t n(j) M n w$

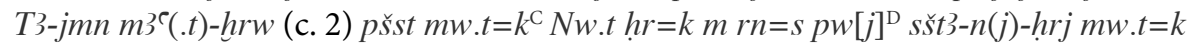
$N w . t r=k m r n[=s]$ (c. 3) $\left[h r j{ }^{\mathrm{E}} d j=s w n n=k\right]^{\mathrm{F}} m n \underline{t} r n n h f . t j . w=k m r n=s m^{\mathrm{G}} n \underline{t} r \underline{h n m}$ $[t]^{\mathrm{H}} s t k m^{-}{ }^{\complement} h[. t n b(. t)](\mathrm{c} .4) \underline{d} w(. t) m r n=s \underline{h n m}(. t)-w r(. t) N w . t s 3[h r s 3(. t)=s] W s j r$ [3s.t-wr.t] [---] (c. 5) $m m s . w^{\mathrm{I}} s b^{\mathrm{J}}$ [sp 2] hr k3=f sb Hrw ${ }^{\mathrm{K}}$ sb Dhwtj sb Dwn- ${ }^{-} n . w{ }^{\mathrm{L}} s b$ Dd.ty $h r k 3=f s b W s j r 3 s . t-w r . t[\ldots]^{\mathrm{M}}$.

Notas editoriales: el texto presenta varios errores, ninguno particularmente raro.

A $s 3$ "proteger" (V16) es usado por similitud funcional (sv:) para $s 3 . t$ "hija" (escrito sólo con el signo G39). La fórmula de filiación $\mathrm{X}$ s3.t (nj) Y ms.n Z "X hija de Y, que Z parió" no sufre modificaciones, por lo que podemos estar seguros de la confusión del escriba. En época ptolemaica V16 vale tanto $s$ como $s 3$ (Daumas, 1995; Leitz, 2004). La escritura refleja la pronunciación contemporánea, donde el masculino $\left({ }^{*} \mathrm{si} 3\right)$ y el femenino ( ${ }^{\star}$ sï3vt) habían quedado igualados $\left({ }^{\star}\right.$ si, por ejemplo, TM Nam 7991 S3.t-3s.t

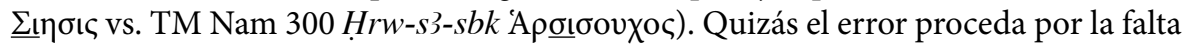
de familiaridad del escriba con el texto, teniendo presente jr $s 3 W$ sjr $\mathrm{N}$ luego de la fórmula de ofrendas en las estatuas llamadas Ptah-Sokar-Osiris en Akhmim (Rindi Nuzzolo, 2017). Variantes de este tipo no son desconocidas en las estelas de esta misma ciudad, por ejemplo la de $T 3$-šr.t-b3st.t preservada en el Oriental Museum en Durham (Nro. 1969, ver Farid, 1995; Munro, 1973), en cuya 1. $6 n k 3 j r-s 3$ Wsjr X (datada en los siglos II-I a.C.).

B No caben dudas sobre la lectura del título sm3.tj en el ataúd de Esoeris. La forma manuscrita del signo - que se ha prestado a confusión con $3 w$ anteriormente - es la usual de este título en época ptolemaica y especialmente en Akhmim, por ejemplo Elias y Mekis (2016), inicios del siglo III a.C., o el ataúd y el cartonnage (manuscritos) 
de Nesmin en el Metropolitan Museum de New York (86.1.50a, b), datado en los siglo II-I a.C.

C Barbash, 2011, paralelo con Stundenwachen V, 53-54. El destinatario del texto es un hombre: véanse los pronombres sufijos de segunda y tercera persona del singular. Descartamos aquí como indicadores de sexo la ortografía errónea de $s 3(. t)$ y la escritura $m$ de $m 3^{\top}(. t)-h r w$, que, como fue explicado, sólo reflejan la pronunciación de la época. Debe tratarse de un error de un escriba menos formado, que introdujo nombres propios y variaciones de espacio en una fórmula que no podía interpretar correctamente. No obstante, debemos señalar como posibilidad la sugerencia que nos realizara el evaluador anónimo $\mathrm{n}^{\circ}$ 2: el sexo masculino podría explicarse por la identificación de la difunta con el dios Osiris. Al respecto, véase (ejemplos más tempranos) Cooney (2008) y Bleiberg (2007); para una revisión de la bibliografía con una opinión contraria, Smith (2017).

D Se lee claramente $p w$ al tiempo que se reconocen trazos del signo $j$. En el ataúd de Nespamay, excepto aquí, $p w j$ se repite luego de $r n=s$ en cada aparición. En nuestro texto, el escriba parece haberlos omitido, probablemente en consideración al espacio.

E Se perdió todo el inicio de la columna pero puede verse la unión de dos trazos correspondiente al signo D21 y parte del signo N1, haciendo segura la reconstrucción del texto.

F Los restos de los signos son mejores que el caso anterior: se observan D37, O 34, E 34, N 35 y V31 sin dificultad, que corresponden al mismo pasaje en el ataúd de Berlin señalado, cuyo propietario se llama Nespamay.

GEn la redacción antigua (TdP) detrás de $m r n=f$ "en su nombre" sigue $n(j)$ "de". Aquí está siempre ausente, excepto (quizás) a inicios de la columna tres. Encontramos $m$ en lugar de $n(j)$, fenómeno bien conocido ya en neoegipcio y que se observa claramente en copto N- "de" que delante de labial > M- (véase Černý y Groll, 1993; Junge, 2005; Layton, 2000).

$\mathrm{H}$ El texto debería decir $h n m=s t w ~ m-$ " "ella te cuida de". El escriba aparentemente no

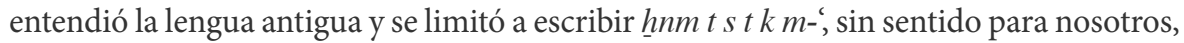
aunque lo traducimos siguiendo lo que el texto debió haber dicho.

I A diferencia del citado ataúd de Nespamay en Berlin, el de Esoeris tiene un texto más cuidado que no omite twt wr jmj ms.w=s "tú eres el grande entre sus hijos" (de la misma manera que pWalters Art Museum 551, Barbash, 2011= $n \underline{t} w(t) s(t) w r \mathrm{jm}$ $m s w=s$ "tú eres, por cierto $[s(t)<s t]$, el grande entre sus hijos"). No obstante sobrevivir $m m s . w$ "entre los nacidos" al inicio de la columna cinco, los signos que se observan al final de la columna cuatro hacen difícil leer el texto previo según la redacción de los Textos de las Pirámides.

J Reconstruimos [ $s p 2$ ], cuyos trazos son observables a la izquierda de $s b$. En esta lectura seguimos la columna 5 in fine del ataúd exterior de Nespamay (Berlín) y Barbash (2011). Sobre esta fórmula, y la aparición de $D w n-{ }^{`} n$.wj y $\underline{D} d . t j$, véase también Barbash (2011).

KEn este y los siguientes dos casos, el escriba omitió $h r k 3=f$. Al igual que en la nota $\mathrm{d}$, es probable que se haya visto apremiado por la ausencia de espacio y haya decidido acortar la fórmula.

LEl escriba agrega un trazo casi horizontal detrás del signo para distinguirlo de $\mathrm{Hrw}$, al que escribe con un trazo vertical (frente a la ortografía habitual de $d w n-{ }^{`} n . w j$, con las alas desplegadas). 
MSolo resta espacio para el determinativo B 1 y $m 3^{\top}(. t)-h r w$.

Traducción: "Palabras dichas por Osiris Esoeris del estolista Nespamay, que parió la Señora de la Casa, Tañedora del Sistro de Min, Taamon, justificada. Que se extienda tu madre Nut sobre ti en este su nombre de 'Secreto del Cielo', tu madre Nut a ti en su nombre de 'Cielo', hace que seas como un dios sin tus enemigos en su nombre de 'Dios'. Te cuida de toda cosa mala en su nombre 'Gran cuenco'. Nut protege a su hija Osiris Esoeris [...] entre los nacidos. Uno fue a su $k a$, Horus fue, Thoth fue, Dewenanwey fue, Djedetifue a su $k a$, Osiris Esoeris fue".

En la parte más baja, entre los pies del ataúd hay texto jeroglífico en tres líneas que lamentablemente no es legible completamente por el depósito de partículas que se le han ido adhiriendo con los años al ser expuesto en posición vertical. Este texto será estudiado en el futuro cercano, aunque podemos adelantar que es claramente formulario. A ambos lados del mismo, se encuentran dos representaciones de Anubis sobre una capilla.

La base o pedestal (conformado combinando tapa y cubeto) está casi completo pues se ha perdido parte de la tabla del flanco izquierdo que corresponde a la tapa. Ésta se encuentra partida a la altura de los tarugos, pero no afectó la decoración ni el texto ahí presente, que fue representado a modo de guarda. El color de base del pedestal es rojo ocre, la guarda es de color amarillo y el texto en negro que se lee en forma continua desde la izquierda del observador (Figura 5 y 6 ):

htp dj nj-sw.t n Wsjr hntj jmntt nț 3 nb $3 b \underline{d} w$ Skr Wsjr hrj jb jpw dj.sn pr-hrww tḥnk.t $k 3 w$ 3pd.w jrp jr.t [...] mrh.t šs mnh.t jh.t $n b(. t) n f r(. t) w^{\ulcorner} b(. t) n k 3 n$ Wsjr $3 s . t-w r . t$.

"Ofrenda que el rey da a Osiris, quien preside Occidente, el gran dios, señor de Abidos, a Sokar, a Osiris que reside en Ipu (= Akhmim), para que den una invocación de ofrendas de pan, cerveza, bueyes, aves, vino, leche, [...], ungüento, alabastro, lino, y toda cosa buena y pura, al $k a$ de Osiris Esoeris".

De la misma manera que el pedestal combina su decoración entre tapa y cubeto, algo similar vemos en la base donde la decoración es una gran $\breve{n}$. Las únicas decoraciones que presenta la cubeta del ataúd son dos serpientes onduladas ocupando todo el lateral a lo largo del mismo (una a cada lado del ataúd) con la cabeza hacia la parte alta, con la corona del Alto Egipto. La parte posterior o reverso del ataúd, tampoco presenta decoración o color.

\section{El nombre de la madre de Esoeris}

Una discusión aparte es el nombre de su madre, puesto que el ataúd se encuentra deteriorado. Castillos (Castillos, 1976, 2004) leyó incorrectamente el título de la madre - posiblemente a causa de la mano cursiva del escriba y la pérdida de la decoración en la zona- como Jnk-n(y)-mnw ("Yo soy de Min", no atestiguado, tanto nombre como ortografía) en lugar del $j h(j . t) n(j) m n w$ "tañedora del sistro de Min”. El título se encuentra escrito con dos ortografías diferentes en este ataúd (véase la inscripción Der. A, más arriba). En este caso, su lectura no presenta dudas, estando bien atestiguada en los diferentes corpora procedentes de esta ciudad, por ejemplo, las estelas Cairo CG 22139 y CG 22067. Para una útil lista de las numerosas variantes ortográficas que pueda presentar este título es aun útil el índice de Kamal (1905).

Brech (2013) observó el error y propuso, luego de leer correctamente el título, la lectura $H r[\ldots]$. La observación directa del ataúd no deja dudas respecto de que el nombre comienza con T3-. Curiosamente, en su visita a Montevideo (4 de Agosto 1936), el egiptólogo belga Jean Capart (copia en el archivo del Centro de Estudios del Egipto 


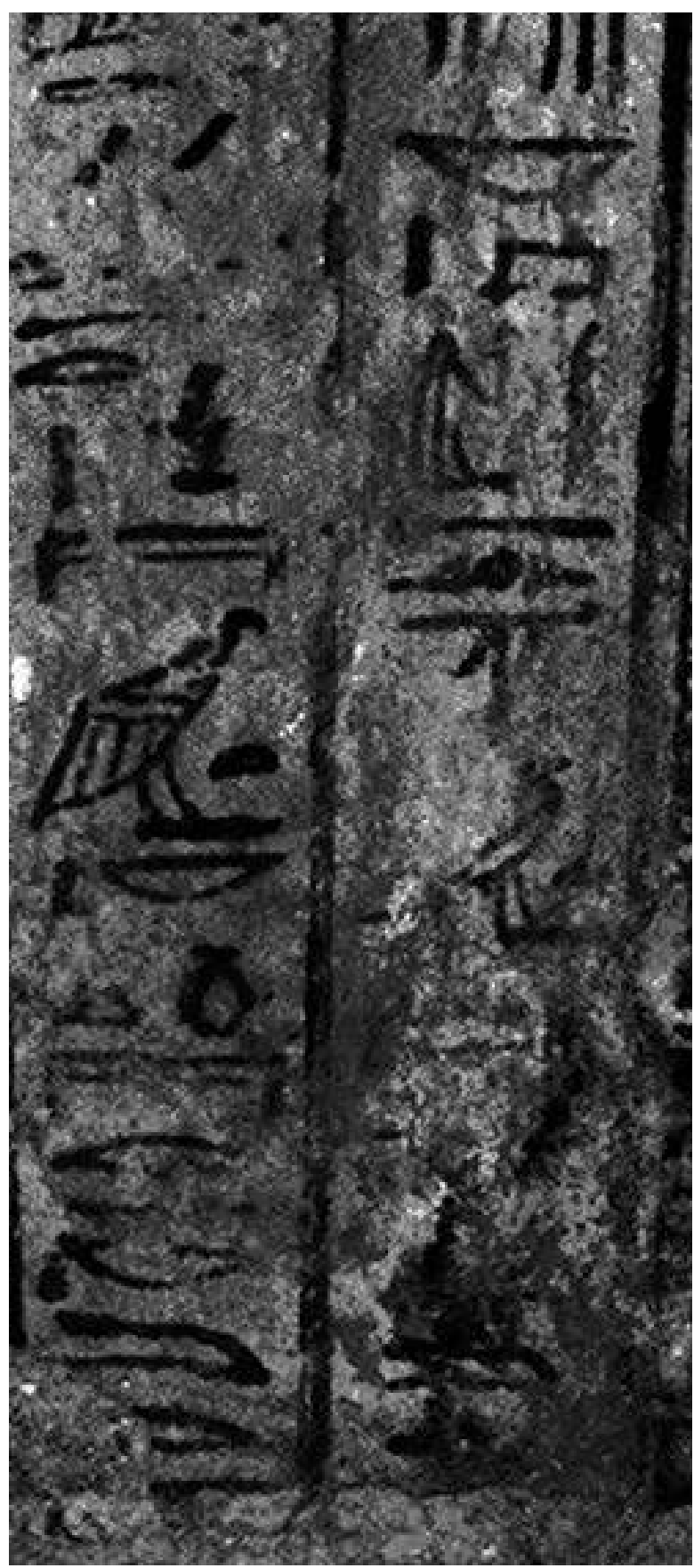

Figura 5. Fin de la columna 1, donde se observa el nombre de la madre de Esoeris. Fotografía Víctor Capuchio.

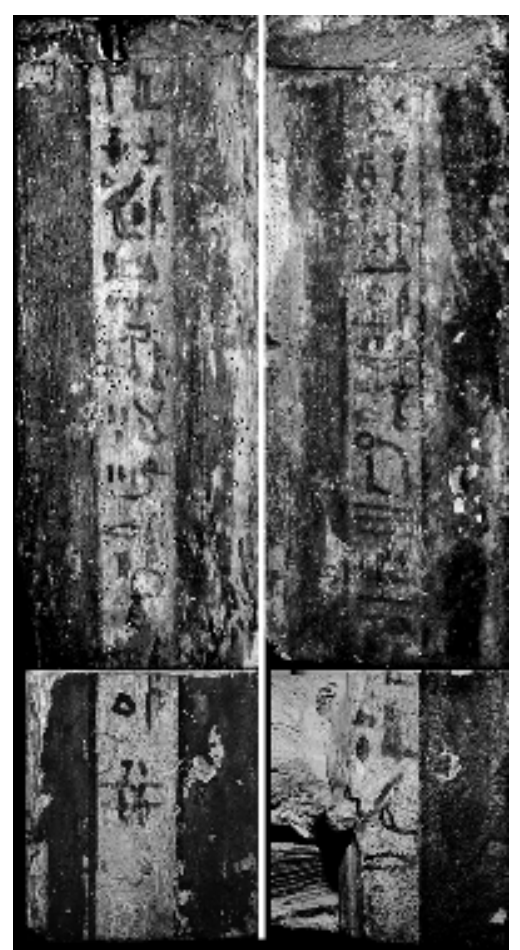

Figura 6. Reconstrucción del texto observado en la Figura s. Fotografía Víctor Capuchio.

y del Mediterráneo Oriental.) asienta en su diario una descripción somera del ataúd, indicando que la madre de Esoeris era tañedora del sistro de Min y su nombre era $\mathrm{Ta}[. .$.$] .$

Afortunadamente, usando el software DStretch de Jon Harman, hemos podido obtener una imagen legible de este sector de la inscripción (véase Figuras 7 y 8), donde se lee claramente $t 3$-jmn.

El nombre T3-jmn TM Nam 7181 (el nombre no fue registrado por Ranke, 1935; algunos ejemplos adicionales, Thirion, 1985, 1995), tal como se encuentra escrito en este ataúd, 


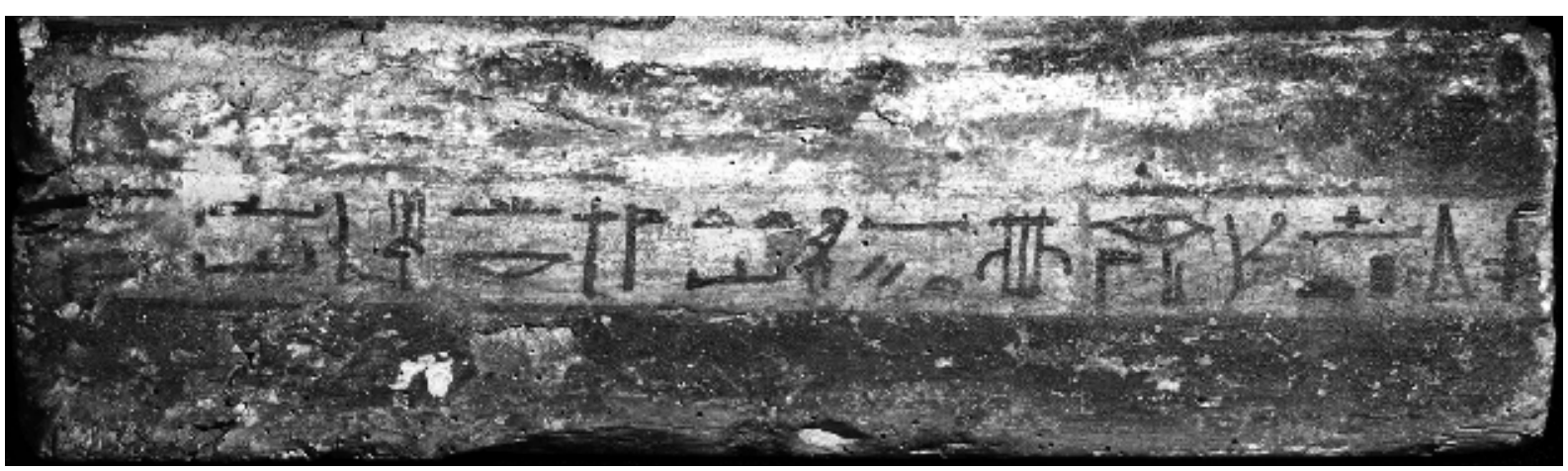

Figura 7. La base o pedestal. Fotografía Víctor Capuchio.
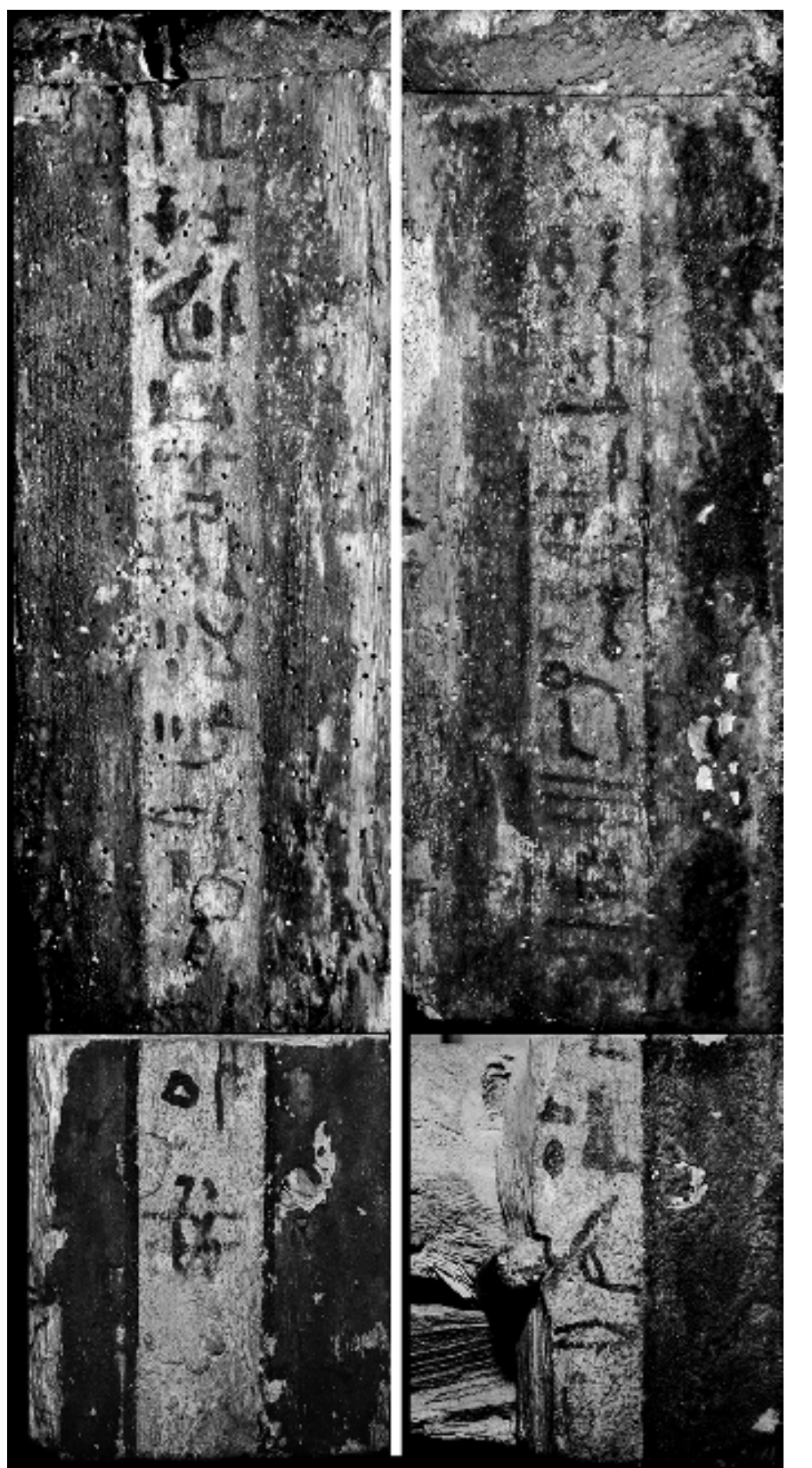

Figura 8. Texto completo de la base, reuniendo el frente y ambos laterales. Fotografía Víctor Capuchio. 
es interesante. Se trata del bien conocido nombre demótico Ta-jmn "la de Amón" (Lüddeckens y Thissen, 1997), cuya retroversión correcta en egipcio clásico debió haber sido $T 3-n(. t)-j m n^{8}$. La otra aparición registrada de T3-jmn procede precisamente de Akhmim: en el conjunto funerario de T3-wsjr [lo mismo, T3-(n(j).t)-wsjr] hija de Ns-mn y T3-jmn, conservado en Michael C. Carlos Museum (Emory: Inv. 2018.010.824A; 2018.010.824B.), previamente en la Senusret Collection. Este fue descripto por Brech (2013) bajo E c 2, datado luego del siglo III a.C., es decir, ambos registrando un uso ortográfico probablemente contemporáneo. En Akhmim, el nombre está registrado cinco veces en demótico, todos ptolemaicos (pero sin relación familiar aparente con el nuestro, ver TM Nam 6975, con referencias).

\section{Conclusiones}

Este trabajo ha presentado una nueva lectura, que creemos correcta, de la inscripción frontal y del nombre y título de la madre de Esoeris, T3-jmn. El dato no es menor por cuanto probablemente permita - en vistas de nueva evidencia prosopográfica que surja en el futuro, tanto de origen epigráfico como papirológico, griego o egipciodatar el ataúd en un momento particular del período ptolemaico y, a partir de esto, datar el conjunto. Este aporte amplía nuestro conocimiento de la prosopografía de las familias de la élite sacerdotal del Akhmim helenístico, a la vez que elimina una persona fantasma (TM Per 162714). Desde la perspectiva de los estudios onomásticos, presenta un nuevo caso en el que el nombre demótico Ta-jmn ha sido transcripto al égyptien de tradition como T3-jmn, lo que demuestra los límites en la formación de este escriba y las características de su trabajo: diversos errores y la reducción de las repeticiones en la fórmula $(p w j ; h r k 3=f)$ probablemente con el fin de adaptarla al espacio disponible.

\section{Agradecimientos}

Al Museo de Historia del Arte (MuHAr) y a su director al momento de escribir este artículo, Gustavo Ferrari. A Carlos Zanola $(\dagger)$ y a Laura Bas. Queremos agradecer también a Marina Escolano-Poveda por sus correcciones y a Andrea Viglione por facilitarnos amablemente el acceso a documentos tanto de su abuelo como de su tatarabuelo conservados en su archivo familiar. Finalmente, queremos agradecer a los dos evaluadores anónimos por sus valiosos comentarios que contribuyeron a la mejora del manuscrito. Cualquier error u omisión, es nuestra exclusiva responsabilidad.
8. Como efectivamente lo encontramos en la estela menfita de $W n-n f r$ h. de $T$ 3-jmn, Copenhagen, Ny Carlsberg Glyptotek AEIN 974, ed. KoedfoedPetersen (1948), Ranke (1935). 


\section{Q Referencias citadas}

»Allen J.P. (2005). The Ancient Egyptian Pyramid Texts. Atlanta: Society of Biblical Literature.

" Assmann, J. (1990). Egyptian Mortuary Liturgies. En S. Israelit-Groll (Ed.), Studies in Egyptology presented to Miriam Lichtheim (Volumen I, pp. 1-45). Jerusalem: The Magness Press, The Hebrew University.

" Assmann, J. (2008). Altägyptischen Totenliturgien, Band 3: Osirisliturgien in Papyri der Spätzeit. Heidelberg: Universitätsverlag Winter.

"Barbash, Y. (2011). The Mortuary Papyrus of Padikakem. Walters Art Museum 551. New Haven: Yale Egyptological Seminar.

"Bianchi, A. (1993). Estudios complementarios sobre la momia egipcia del Museo Nacional de Historia Natural de Montevideo. Montevideo: Publicaciones de la Sociedad Uruguaya de Egiptología.

"Bleiberg, E. (2007). The Coffin of Weretwahset/Bensuipet and "Scribal Errors" on Women's Funerary Equipment. Bulletin of the Egyptological Seminar, 17, 35-44.

"Bracco, R. y Ures, C. (2002). Informe sobre las edades $\mathrm{C}_{14}$ de las muestras tomadas a la momia egipcia del Muhar. Informe al Museo de Historia del Arte. Manuscrito inédito.

» Brech, R. (2013). Spätägyptische Särge aus Akhmim. Eine typologische und chronologische Studie. Gladbeck: PeWe Verlag.

" Capuchio, V. (2004). Proyecto Gran Isis. Montevideo: Museo de Historia del Arte.

"Capuchio, V. (2016). Informe preliminar de la tomografía realizada a la momia del Museo de Historia del Arte de Montevideo. Montevideo: Museo de Historia del Arte.

"Castillos, J. (1976). A late Egyptian mummy at the National Natural History Museum of Montevideo. Revue d'Égyptologie, 28, 48-60.

"Castillos, J. (1980). Una momia egipcia de la Baja Época conservada en el Museo Arqueológico Palacio Taranco de Montevideo. Montevideo: Ediciones Maat.

" Castillos, J. (2001). Eso-Eris, the Wandering Mummy. En H. Györy (Ed.), Mélanges offerts à Edith Varga (pp. 29-36). Budapest: Musée Hungrois des Beaux-Arts.

"Castillos, J. (2005). Further Remarks on the Montevideo Mummy. Journal of the Serbian Archaeological Society, 21, 343-348.

» Černỳ, J. y Groll, S. (1993). A Late Egyptian Grammar. Fourth Edition. Roma: Editrice Pontificio Instituto Biblico.

" Cockitt, J. A., Martin, S. O. y David, R. (2014). A new Assessment of the Radiocarbon Age of Manchester Mummy No. 1770. Yearbook of Mummy Studies, 2, 95-102.

»Cooney, K. (2008). The Problem of Female Rebirth in New Kingdom Egypt: The Fragmentation of the Female Individual in her Funerary Equipment. En C. GravesBrown (Ed.), Sex and Gender in Ancient Egypt (pp. 1-25). Swansea: The Classical Press of Wales.

»Daneri, A. (1981). El Dr. Dardo Rocha y las piezas egipcias del Museo de Ciencias Naturales de La Plata. La Plata: Museo Dardo Rocha.

"Daumas, F. (1995). Valeurs phonétiques des signes hiéroglyphiques d'époque gréco-romaine. Montpellier: Université de Montpellier. 
” de Meulenaere, H., Limme, L. y Quaegebeur, J. (1985). Peter Munro, Die spätägyptischen Totenstelen: Index et Addenda. Bruselas: Musées Royaux d'art et d'histoire.

»Elias, J. (1993). Coffin inscription in Egypt after the new kingdom: a study of text production and use in elite mortuary preparation (Tesis Doctoral inédita), University of Chicago, Estados Unidos de América.

»Elias, J. y Lipton, C. (2008). The Social Parameters of Mummification in Akhmim. En P. Atoche, C. Rodríguez Martín \& M. A. Ramírez Rodríguez (Eds.), Mummies and Science (pp. 507-514). Santa Cruz de Tenerife: Academia Canaria de la Historia.

»Elias, J. y Mekis, T. (2016). The yellow-on-black coffin of the oracle scribe Hor in the Swansea Museum. Chronique d'Égypte, 91(182), 227-263.

" Farid, A. (1995). Fünf demotische Stelen aus Berlin, Chicago, Durham, London und Oxford mit zwei demotischen Türinschriften aus Paris und einer Bibliographie der demotischen Inschriften. Berlin: Achet Verlag.

» Galan, J. M. (2013). Nut on the Ceiling of the Burial Chamber of Djehuty (TT II). En E. Frood y A. McDonald (Eds.), Decorum and Experience. Essays in Ancient Culture for John Baines (pp. 119-126). Oxford: Griffith Institute.

» Gauthier, H. (1931). Le personnel du dieu Min. El Cairo: IFAO.

» Grimm, G. (1974). Die römischen Mummienmasken aus Ägypten. Wiesbaden: Harrassowitz.

» Junge, F. (2005). Late Egyptian Grammar. An Introduction. Second Edition. Oxford: Griffith Institute.

》 Kamal, A. (1905). Stèles ptolémaiques et romaines. El Cairo: IFAO.

"Layton, B. (2000). A Coptic Grammar with Chrestomaty and Glossary. Sahidic Dialect. Wiesbaden: Harrassowitz Verlag.

» Leitz, Ch. (Ed.) (2002). Lexikon der ägyptischen Götter und Götterbezeichnungen, Band 1. Leuven: Peeters.

» Leitz, Ch. (Ed.) (2002b). Lexikon der ägyptischen Götter und Götterbezeichnungen, Band 7. Leuven: Peeters.

»Leitz, Ch. (2004). Quellentexte zur ägyptischen Religion I: Die Tempelinschriften der griechisch-römischen Zeit. Münster: Lit Verlag.

» Loprieno, A. (1995). Ancient Egyptian. A Linguistic Introduction. Cambridge: Cambridge University Press.

» Lüddeckens, E. (1980). Demotisches Namenbuch. Band I, Lieferung 1. Wiesbaden: Dr. Ludwig Reichert Verlag.

" Lüddeckens, E. y Thissen, H.-J. (1997). Demotisches Namenbuch. Band I, Lieferung 15. Wiesbaden: Dr. Ludwig Reichert Verlag.

" Miatello, L. (2012). Ptolemaic Mummy Masks with Spells from the Book of the Dead concerning the Head. Journal of the Society for the Study of Egyptian Antiquities, 39, 51-85.

" Mosher, M. (2001). The Papyrus of Hor (BM EA 10479) with Papyrus Mac Gregor: The Late Period Tradition at Akhmim. Londres: British Museum.

" Mosher, M. (2002). The Book of the Dead Tradition at Akhmim in the Late Period. En A. Egberts, B. P. Muhs y J. van der Vliet (Eds.), Perspectives on Panopolis (pp. 201-209). Leiden: Brill.

" Mosher, M. (2016). The Book of the Dead, Saite through Ptolemaic Periods. A Study of Traditions Evidente in Versions of Texts and Vignettes, Volume 1, BD Spells 1-15. Prescott: SPBD Studies. 
» Munro, P. (1973). Die spätägyptischen Totenstelen. Glückstadt: J. J. Augustin.

»Nicholson, P. T. y Shaw, I. (2000). Ancient Egyptian Materials and Technology. Cambridge: Cambridge University Press.

» Ranke, H. (1935). Die Ägyptischen Personennamen. Vol. I. Glückstadt: Verlag von J. J. Augustin.

»Rindi Nuzzolo, C. (2017). Tradition and Transformation. Retracing Ptah-Sokar-Osiris figures from Akhmim in Museums and Private Collections. En T. Gillen (Ed.), (Re) productive Traditions in Ancient Egypt (pp. 445-447). Liège: Presses Universitaires de Liège.

» Ritner, R. K. (2016). King Petemenekh: New Kingdom Royal Sarcophagi Texts on a Private Coffin. En K. Ryholt y G. Barjamovic (Eds.), Problems of Canonicity and Identity Formation in Ancient Egypt and Mesopotamia (pp. 179-200). Copenhagen: Museum Tusculanum Press.

»Salem, L. (2018). El orientalismo en tensión: lecturas sobre Egipto en los relatos de viaje de fines del siglo XIX en Argentina. Revista de Historia Americana y Argentina, 52(2), 39-81.

"Santos, D. M. (2003). La colección egipcia del Museo Etnográfico I. Aegyptus Antiqua, 11, 42-64.

"Santos, D. M. (2012). Las momias egipcias del Museo de La Plata. Estudios recientes. Aegyptus Antiqua, 13, 60-69.

"Scalf, F. (2014). Passports to Eternity. Formulaic Demotic Funerary Texts and the Final Phase of Egyptian Funerary Literature in Roman Egypt. (Tesis Doctoral inédita), The University of Chicago, Estados Unidos de América.

»Scalf, F. (2017). Book of the Dead. Chicago: Oriental Institute.

"Smith, M. (2017). Following Osiris. Perspectives on the Osirian Afterlife from Four Millennia. Oxford: Oxford University Press.

»Stadler, M. A. (2001). Ein 'vollkommenes Gesicht' aus dem spätptolemäischen Ägypten. En M. Maier y St. Specht (Eds.), Blickwechsel (pp. 145-164). Würzburg: Ergon-Verlag.

" Thirion, M. (1985). Notes d'onomastique. Contribution à une révision de Ranke PN [Quatrième série]. Revue d'Égyptologie, 36, 125-143.

» Thirion, M. (1995). Notes d'onomastique. Contribution à une révision de Ranke PN [Dixième série]. Revue d'Égyptologie, 46, 171-186.

»Vergote, J. (1961). Sur les mots composés en égyptien et en copte. Bibliotheca Orientalis, 18(5-6), 208-214.

"Vernus, P. (1978). Athribis. Textes et documents relatifs à la géographie, aux cultes et à I'histoire d'une ville du Delta égyptien à lépoque pharaonique. El Cairo: IFAO.

» Viglione, L. A. (1890). Cartas de Nápoles, Alejandría y Cairo de Egipto. Buenos Aires: Imprenta de Martin Biedma. 